\title{
1 Adolescence and Families
}

In this book we aim to explore the research that has emerged over the last couple of decades about adolescents within the family. The primary focus is on adolescence in the western world as that is our experience and knowledge, and where most of the research is carried out. We have aimed, however, to incorporate cross-cultural information, whilst not claiming to be experts in this vast and complex area. In the current chapter we aim to give a general overview of how adolescence is viewed and some of the more recent societal changes that have impacted on the adolescent experience. We also discuss the links between adolescence and family life, the main focus of this book.

In this chapter we will discuss views of adolescence and set the context for understanding the adolescent experience in this modern world. We will discuss adolescence as a life stage, and explore the chronology of adolescence from childhood to adulthood. We acknowledge that all families are not the same and that adolescents are likely to have different experiences depending on the nature of the family in which they develop. We also give a brief overview of current research on adolescent brain development and implications for adolescent lifestyle and behaviour.

\subsection{Views of Adolescence}

The view that adolescence is a time of storm and stress, both for the adolescents themselves and for their families, has lost dominance, although it is still true that for some adolescents, this is a challenging stage of their lives. The notion that adolescence is a stressful time in families comes from evidence that indicates there are several areas where difficulties for families can arise. During this time there can be increased conflict with parents (see Chapter 4), and adolescents can become more moody and higher in risk-taking (Arnett, 2007a). As adolescents seek new experiences they are likely to be exposed to various potentially problematic or dangerous influences (see Chapter 6).

The three main developmental tasks for adolescents are (1) to become independent from their parents, (2) to develop an individual identity and (3) to develop mature peer relationships and explore romantic relationships whilst maintaining strong connections with parents (Arnett, 2000, 2001). (These issues of individuation and identity development will be discussed in more detail in Chapter 2.) Young people also need to prepare themselves, through education and training, for the world of work. It is important to remember that these tasks are no longer seen as peculiar to adolescence, but as continuing to be developed during emerging adulthood. (See Chapters 2 \& 4)

Elliot and Feldman (1990) refer to adolescence as a time for self-discovery and for learning the skills that are needed for adulthood. Adolescents are developing new 
ways of thinking that can change the ways in which they see themselves and their family members (Larson \& Richards, 1994; Sroufe, Egeland, Carlson, \& Collins, 2005). The fact that adolescents have generally developed advanced thinking skills, allows them to reflect upon and question existing family conventions (Grotevant \& Cooper, 1986; Sroufe et al.). This questioning, of course, may contribute to increased conflict within the family; however, conflict can be a way for adolescents to learn how to deal with differences of opinion and negotiate an individual point of view.

Families vary in their ability to cope with change and to accept adolescents' strivings for individuality; however, the need for flexibility in the family becomes more important when children reach adolescence (Hauser, Powers, \& Noam, 1991). The amount of disruption created by adolescents' autonomy-seeking will be at least partly dependent upon the parents' levels of acceptance or resistance to this process (Noller, 1995; Noller \& Patton, 1990). Strong parent-adolescent relationships that manage the changes in the ways parents and adolescents relate to one another, are likely to support adolescents' abilities to explore relationships outside the family (Collins \& Laursen, 2004).

In contrast to the play-friends children have, intimacy in friendships becomes a feature for adolescents, although girls tend to have more intimacy in friendships than do boys (Arnett, 2007a). The friendships that adolescents have involve trust, loyalty and shared values (Steinberg, 2005). Such friendships provide young people with opportunities to develop their social skills.

Young people learn about relationships within the family and this experience provides them with the basis for exploring and expanding their social skills within peer friendships (Sroufe et al., 2005). Adolescents tend to report that their relationships with friends and romantic partners are closer than their relationships with family members. This focus on peers does not mean that parents are not important to young people. In addition the quality of relationships with peers and parents is linked; adolescents who enjoy good relationships with parents are more likely to have good relationships with their peers (Steinberg, 2005).

Just as friendships do, romantic relationships become an increasingly important part of adolescents' lives (Brown, 2004). It appears that adolescents may model their dating relationships on what they have seen in the relationship between their parents (Steinberg, 2005). Thus adolescents whose families have poor relationships and relationships that are highly conflicted are less likely than those from harmonious families to learn the skills necessary for successful relationships outside the family. In addition, the quality of adolescents' relationships with both parents and peers influences the quality of their romantic relationships (Brown, 2004). Early serious dating before the age of 15 appears to be associated with poorer social development, especially for girls. Later dating, and dating that occurs in the context of supportive family relationships and good peer friendships, can have a beneficial effect on adolescents' self-esteem (Steinberg, 2005). Therefore, the development of 
relationships within the family also has implications for the timing and quality of future relationships.

We want to emphasise that we view adolescence as a dynamic concept interdependent with the complex and changing world in which we live. The ways in which adolescence is defined varies with social changes in any given place and between cultures. Therefore, the many changes in our world over the last couple of decades have impacted on both views of adolescents and how their development is understood, as well as on the experiences of adolescents themselves.

Given that our primary focus is on families, it is important to acknowledge that not all families are the same, with many variations in family structure. What we generally call the nuclear family is traditionally seen as involving two married parents and their biological child or children. Variations include two unmarried parents (cohabiting or de facto) and their biological children, a single parent (generally the mother) and her biological children to one or more fathers, a biological parent and his or her child or children and a stepparent. Families may also include adopted children or children created by artificial insemination, including donor sperm or donor egg. In families involving gay parents, there are two mothers or two fathers, either or both of which may be unrelated biologically to the child. There are also families where children live with foster parents due to the inability of any biological family member to provide care.

Some families have more contact with the members of their extended family than others, and are more open to influence from family members such as grandparents. Some children are even raised by grandparents because of the inability of the parents to fulfil the parenting role. Reasons that parents may be unable to care for their own children might include incarceration, drug or alcohol dependency, mental or physical health issues or death. In addition, in some cultural groups, particularly those from collectivist cultures, grandparents are likely to be co-resident with the family.

Obviously, covering all of these different types of families in this book is impossible. In addition, for some types of families there is a great deal of research and for other types there is very little. Our areas of knowledge direct our focus towards the more predominant types of families: nuclear families, the extended family, singleparent families, and divorced and remarried families.

\subsection{Stereotypes of adolescence}

The notion of storm and stress in adolescence is really a stereotype, an exaggerated image of adolescence based on the behaviour of a few (Arnett, 2007b). Arnett acknowledges that although there may be some truth to the negative stereotypes of adolescence, there are clearly differences between individuals and between cultures in the extent to which adolescence could be seen as stormy or stressful (Arnett, 1999). 
The National Association for Social Workers in Washington put out a practice update paper in 2002 that discusses how negative stereotypes about adolescence in our communities are more dominant than positive ones. They argue that a negative social view is damaging to the well being of adolescents in our communities as it can lead to adolescents taking on board the image that is portrayed of them, which, in turn, affects their own self-image, what social psychologists call self-fulfilling prophecies. These social workers concluded that promoting positive images of adolescents would break down the negative stereotypes, and provide a more realistic account of their behaviour (NASW, 2002).

We believe that a balanced approach is important. Researchers and practitioners cannot ignore the potential for adolescents to make poor decisions and to engage in negative behaviours. On the other hand, it is totally inappropriate for all adolescents to be labelled as dysfunctional because of the bad behaviour of a small minority. We will discuss the problems that adolescents can confront during this stage of life. (See Chapter 6)

A framework has been put forward in reaction to this focus on the negative aspects of young people's behaviour. This framework has been called Positive Youth Development (see Chapter 2) and has been suggested as a way to view adolescence from an asset- or strengths-based perspective that acknowledges young people as agents in their own lives (e.g., Benson, Scales \& Mannes, 2003; Damon, 2004; Klatt \& Enright, 2009; Larson 2000; 2006; Lerner, Brentano, Dowling, \& Anderson, 2003).

However, stereotypes of adolescence abound in our communities and within some families. Adolescents are perceived by many adults as rude, irresponsible and wild. Many adults have little contact with the adolescents in their communities and this lack of contact can contribute to an acceptance of negative stereotypes. If communities are more connected, and the adults and adolescents experience their community this way, adults tend to have a more positive view of adolescents (Zeldin \& Topitzes, 2002). However, adults can tend to hold negative stereotypes of adolescents even when they are not consciously aware that they do (Gross \& Hardin, 2007).

In a longitudinal study conducted over five years, adolescent participants were initially assessed from Grade 7 and then reassessed at Grades 10 and 12. Jacobs and colleagues (Jacobs, Chhin \& Shaver, 2005) assessed parents' beliefs in negative stereotypes of adolescence in general, and the link between those stereotypes and their views of their own adolescent. Parents who held stronger stereotypical beliefs about adolescence viewed their own adolescent as more peer-focused and concerned about their social life than did parents whose beliefs in stereotypes were weaker. This finding held true over a three-year period. So stereotypes can even impact parents' views of their own children whom they know as individuals. Also, and even more concerning, was that parents' beliefs were related to the later behaviour of their children, and to their relationships with them. When their parents' stereotypical beliefs were strong, adolescents were more focused on peers, displayed more 
problematic behaviour and had poorer relationships with parents. This study also indicated that having at least one parent who did not hold strong stereotypical beliefs was associated with less negative outcomes for adolescents.

\subsection{The Chronology of Adolescence}

The steps required to move from adolescence into adulthood have undergone significant changes for many adolescents over the last 50 years (Arnett, 2007a) as has our understanding of this developmental stage. The chronology of adolescence is dictated, to an extent, by societal needs (Sisson, Hersen \& Van Hasselt, 1987; Thurlow, 2005). In the current context of a diminishing need for younger people in the work force, and the trend toward longer education and dependence upon parents, adolescence could be seen as lasting until the early to mid twenties, rather than to the age of 18. Because of these changes, the position of adolescents is somewhat ambiguous, as the extended duration of adolescence means that expectations of both parents and adolescents for the adult status of the adolescents are delayed (Newton, 1995; Thurlow, 2005). Previously parents and their children could expect a fairly consistent chronology from childhood to adulthood and independence. Now, to a much greater extent, this trajectory is more variable and adolescents have to rely on the family for longer periods, with implications for the adolescents, their parents and other family relationships.

\subsubsection{Childhood to Adolescence}

An important principle to understand is the significance of developmental history to how young people cope with adolescence and to the quality of family relationships during this "challenging period" (Sroufe et al., p.175). These authors, based on a longitudinal study of American children beginning before birth and continuing for 30 years, discuss "the legacies of the earliest years of life" (Sroufe et al., p.175), showing very clearly the ways that later development builds on earlier experiences in the family and beyond. For example, the ability to regulate emotion and the sense of inner worth and ego-resiliency are seen as products of earlier developmental history. These attributes are critical to the acquisition of the skills needed for positive relationships within the family and to later social relationships within the broader social world. As Sroufe et al. point out "more children have problems, and more serious problems, during adolescence than during middle childhood" (Sroufe et al., p.175), problems that they see as resulting primarily from developmental history. Steinberg and Morris (2001) also provide support for this viewpoint. In other words, problems in adolescence do not come out of nowhere, but build on the foundations established in infancy and childhood. 
It is important to acknowledge that families participating in the Sroufe et al. (2005) study were poor families that would be considered at risk for developmental problems. Nevertheless, many of these families and their young people developed in positive ways. Hence it is clear that some families that would normally be considered vulnerable can transition effectively through this phase and actually thrive because of their positive connections and supportive family contexts.

In fact, there is a great deal of evidence that most adolescents do not develop significant problems and do cope effectively with the challenges they confront during this period of their lives (Arnett, 1999; Steinberg, 1999; Steinberg \& Morris, 2001). Gutman and Eccles (2007) showed that those adolescents whose family environments satisfy their developmental needs such as providing appropriate involvement in decision-making are more likely to become well-adjusted young people. Adolescents' beliefs about their choice to be involved in decision-making with their parents, or make decisions on their own certainly seem important to positive development (Van Petegem, Beyers, Vansteenkiste \& Soenens, 2013; Van Petegem, Vansteenkiste \& Beyers, 2013).

As Parke (2004a) notes there is a very strong interdependence among family members. Hence, to understand families and their relationships, we need to realise the interconnections among the roles and functions of various members and how they affect each other. Thus as children move into adolescence, where they have an expectation of increasing independence, parents begin to change from being advisors to being guides, gradually allowing more autonomy to their young people. In an earlier study, Grotevant and Cooper (1986) showed the need for this change in family relationships so that adolescents can develop more equitable relationships with their parents.

\subsubsection{Early Adolescence to Late Adolescence}

Changes between early and late adolescence occur for a number of reasons. For example, the onset of puberty, cognitive development, transitions to high school, and changing relationships with peers and family members (Gutman \& Eccles, 2007). For some adolescents, these changes are constructive and are likely to promote positive growth and adjustment. For other adolescents, however, these changes are likely to have an impact on both their self-esteem and their mental health. Further, for a minority of adolescents, these changes are likely to increase the risk of their becoming involved in a range of problem behaviours (Bongers, Koot, Van der Ende, \& Verhulst, 2004; Eccles, Midgley, Wigfield, Reuman, et al., 1993; Hankin, Abramson, Moffitt, Silva, McGee, \& Angell, 1998). (See Chapter 6) The impact of these changes may also vary by gender and ethnicity.

Much has been made of the lack of rites of passage for adolescents coming into adulthood (Hendry, Glendinning, Shycksmith, Love \& Scott, 1994; Newton, 1995), and the ambiguity surrounding adolescents' status (Thurlow, 2005). These issues are emerging as increasingly complex, and researchers have looked to new ways to 
understand early life transitions. A clear pathway from childhood to adolescence to adulthood no longer represents the experiences or expectations of most adolescents and their families.

Because of this complex context in which adolescents make the transition through to adult status, the concept of "emerging adulthood" has been used to describe a newly-defined developmental phase encompassing the ages 18-25 years, a period of transition between adolescence and adulthood (Arnett, 2000a; 2004; 2007). According to Arnett (2007) it is during this period, in western societies at least, that young people are finalising the tasks they began in adolescence and defining their individual identities and their futures in love and work. Emerging adults are moving beyond reliance on their parents but are not quite at a stage where they have made commitments to partnerships or careers.

\subsection{The Developing Brain}

Developmental neuroscience, and in particular the use of Functional Magnetic Resonance Imaging (fMRI), has enhanced current knowledge of brain development and provides information that has implications for our understanding of adolescence. This research indicates that brain development is not complete in adolescence and that the pre-frontal cortex is changing and developing until the mid twenties (Keating, 2004). This plasticity of the brain during adolescence is helpful for learning and as we learn through new experience, adolescents are often seen as "sensation seeking". For this reason, these new experiences, both positive and negative, will impact upon development (Konrad, Firk \& Uhlhaas, 2013). Adolescent brain development and restructuring, like behavioural changes, are related to the hormonal changes of puberty (Goddings, Heyes, Bird, Viner \& Blakemore, 2012; Konrad et al., 2013). Therefore it is fair to say that adolescents have many changes to contend with whilst they mature physically and emotionally in an ever-changing, and more ambiguous world.

Different parts of the brain develop at different rates, and during adolescence there is a lot of growth in the pre-frontal cortex. This development involves the higher functioning areas of the brain and is about thinking and psychosocial skills that are related to the ability to manage emotions and plan and make decisions, among other things (Steinberg, 2004). In a review of the literature on brain development, Konrad and colleagues (2013) indicate that the imbalance between the earlier maturing limbic and reward systems of the brain and the later maturing pre-frontal control system of the brain in adolescence may underlie adolescent emotional reactivity and risky behaviour. Steinberg (2004) and Konrad and colleagues (2013) indicate that the advances in neuroscience have supported the view that adolescence is a time of development that holds both risks and opportunities for young people. These findings underline the importance of good quality family relationships that provide positive guidance and support to adolescents until their brains are fully developed. 
It was once thought that adolescents participated in risky behaviours (e.g. drug use, unprotected sex, dangerous driving) because they lacked the knowledge and skills to make good decisions about these issues. However, current knowledge provides evidence that adolescents understand the hazards involved in such risky activities but because of their lack of decision-making skills, decide to engage in them regardless (Steinberg, 2007). Steinberg contends that the less-developed psychosocial brain-pathways of adolescents may prevent them from making good decisions in some of these situations. The social advantages of risky behaviour may be considered by adolescents to be more advantageous than the risks themselves (Konrad et al., 2013). In other words, when the socio-emotional part of the brain is active, such as when adolescents are with peers, the brain processes that provide control are less likely to be able to exert an influence on decision-making, and adolescents are more likely to make bad or risky decisions. Obviously if adolescent brains are also fuelled by the use of alcohol and other mind-altering substances, disaster is possible (see Chapter 6).

Steinberg (2007) also argues that understanding the environment, such as family and peer relationships, together with the ways the development of neural pathways affects adolescents' abilities to make good decisions and manage their emotions, is important. It will be interesting to see the advancements in these fields of research over the next decade and hopefully this branch of science will continue to be consistent with developmental research.

\subsection{Community and Political Involvement}

Studies of the benefits of community involvement for young people show that those who are active in their communities have lower rates of the kinds of problem behaviours in which adolescents can become involved (Allen, Philliber, Herrling \& Gabriel, 1997; Barber, Eccles \& Stone, 2001; Mahoney \& Cairns, 1997).

In a study of adolescents in their final years of secondary school, and using measures of psychological adjustment, identity development, and parent and peer interactions to predict their community and political involvement, four distinct groups of adolescents were identified (Pancer, Pratt, Hunsberger \& Alisat, 2007). The researchers labelled the four groups as Activists, Helpers, Responders and Uninvolved. Activists tended to be involved in both community and political activities; Helpers helped people in their communities on an individual basis but tended not to be involved in political activities; Responders did not initiate helping or political activities but were willing to volunteer assistance when that was needed; the fourth group were the uninvolved adolescents.

Involved adolescents, whether activists, helpers or responders, reported higher levels of optimism, self-esteem and support than the uninvolved adolescents. The authors point out that the sample was not randomly selected, although it was representative of a significant proportion of the young students in the area. The authors 
also acknowledge that the direction of causation needs to be interpreted with caution. It is possible that it is the young people who are better adjusted who are the most likely to get involved in their communities, rather than that community involvement leads to better adjustment. Realistically the effects could be bidirectional.

\subsection{Summary}

The ways in which 'adolescences' are defined varies across cultures and with the changes in societies. Currently, adolescents have greater freedom but fewer structures that support them to make choices and decisions about their futures. The view that adolescence is a stressful time for families has lost dominance. Most families make the transition through this stage without any major disturbance.

Stereotypes of adolescents are largely negative and many adults perceive adolescents as rude, irresponsible and wild. When adults and adolescents are connected with each other and their communities, adults tend to view adolescents more positively. Adults are not always consciously aware of the stereotypical views they hold of adolescents. Parents who hold strong stereotypical views of adolescents in general can perceive their own adolescent in a more negative light than those who do not hold such strong stereotypes. Also, when parents hold strong stereotypical views, their adolescents are likely to display more problematic behaviour and have poorer relationships with them.

New discoveries in neuroscience have indicated that brains are not fully developed until the mid-twenties. This later development is about the refinement of thinking and psychosocial skills. In adolescence the pathways in the brain that provide control are less developed and adolescents may be more likely to make risky decisions, particularly when they are with their peers.

In Chapter 2 we explore some of the many theoretical perspectives that are used to explain and understand this life stage. We focus on Family Systems Theory, cognitive theories, Separation-Individuation, identity formation, Attachment theory, and Life Course Theory. We also consider the relatively new emphasis by some scholars of Positive Youth Development.

In Chapter 3 we focus on the diverse environments in which young people grow up, and the impact of those environments on the well-being and development of adolescents. We look at the effects of family structure, financial difficulties, parent characteristics, issues related to parenting such as parental mental health and the quality of the marital relationship. A further focus is on the importance of relationships with siblings and the extended family and the communities with which the adolescents are involved.

In Chapter 4, we explore the quality of family communication and relationships. The primary focus is on the implications of these processes for adolescent adjustment. We focus on the changing nature of these relationships as adolescents renegotiate 
roles and rules with their families, to ensure that their relationships reflect the increasing need for adolescents to become independent. We include discussion of conflict, decision-making, disclosure and secrecy, discipline, sibling communication and families' use of technology as a communication tool.

The impact for children of parental divorce in the western world has featured in much research over the last few decades and we explore the impact of family breakdown on adolescents in Chapter 5. We discuss the impact of common living arrangements for children following the divorce of their parents, and communication, conflict and parental disclosure following the breakup. We also address the long-term effects of family breakdown on adolescent adjustment and later relationships, and the ways that adolescents manage their relationships with the non-resident parent and siblings. Another focus is the impact of living in a stepfamily.

In Chapter 6 we look at a broad array of adolescent issues and the multiple features of families that can contribute to adolescent outcomes. We explore the issues in terms of externalising and internalising behaviour. We discuss alcohol and other drugs, issues around sexuality, antisocial behaviour and delinquency, depression, self-harm and suicide, and eating disorders.

Whilst leaving home during adolescence is not the norm for most young people many do leave for a variety of reasons. In Chapter 7 we aim to give an overview of the contexts in which adolescents may leave the family home at this early stage of life. We consider the impact of family structure, and the quality of family relationships on the timing of leaving home for young people. We also discuss other reasons for leaving home such as for study or career options, or to cohabit or marry. We also focus on young people who run away and those who become homeless.

In Chapter 8 we focus on the concept of resilience and explore both the causes and consequences of resilience, asking why some adolescents are able to handle adversity so well and others not so well. We also discuss the complex links between parenting and temperament, focusing on some longitudinal research, some of which was initially carried out on infants and children, following these children into adolescence and beyond.

Adolescents are living in a rapidly changing world that impacts the ways in which this developmental stage is viewed and studied. The ways that society and the world in general have changed over the last few decades have created new challenges and complexities for both adolescents and their families. For this reason support, guidance and understanding from parents are critical to the well being of adolescents, during this time and into adulthood. In the next chapter we explore the theories that are used to understand and investigate the adolescent experience in the context of the family. 